\title{
Are the combined oral contraceptive pills needed for management of the simple ovarian cysts in reproductive women? (Randomized controlled study)
}

S.A.El beree, K.M.Salama, S.T.Mostafa and A.S.Mohamed

Obstetrics and gynecology, Dept., Faculty of Medicine, Benha Univ., Benha, Egypt

E-mail:ebaidahmed68@gmail.com

\begin{abstract}
Background: Functional ovarian cysts (follicular and corpus luteum) are a common gynaecological problem among women of reproductive age worldwide. The aim of the present study was to compare the different modalities of functional ovarian cyst management i.e. expectant management and combined oral contraceptive pills (C.O.P) to know if combined oral contraceptive pills are needed in the management of functional ovarian cysts in reproductive aged women. Methods: One hundred eighteen patients with a probably functional ovarian cyst based on ultrasound criteria (unilateral, 2.5-6 centimetres in diameter, thin wall, unilocular without internal echoes and no solid parts) were randomly allocated into two groups after giving informed consent: Group A: women who will be managed expectantly. Group B: women who will be given combined oral contraceptive pills. Women managed by either of the two modalities (Group A and Group B) will be followed up by transvaginal ultrasound monthly for three successive months to evaluate the size, and location of the functional ovarian cyst. Results: At the beginning of the study, the two groups were comparable with respect to age and ultrasound criteria of their functional ovarian cyst we found after 3 months remission in 46 (78.0 percent ) women in group (A) and50 (84.7 percent ) women in group (B). Conclusion: There is no proven advantage in the use of combined oral contraceptives.
\end{abstract}

Keywords: Contraceptive, Pills, Ovarian, Cysts, Reproductive.

\section{Introduction}

A simple ovarian cyst is a fluid-filled sack, also known as a functional cyst, in the ovary. They often do not produce any symptoms. Sometimes they may inflate, cause lower stomach discomfort or cause lower back discomfort. If the cyst either bursts open or causes significant ovarian discomfort to twist. This may lead to vomiting or fainting. The majority of cysts are inoffensive. Every month, most reproductive women produce tiny cysts. Approximately 8 percent of women have large kysts that create difficulties before menopause. Ovarian cysts are most likely to be cancer in approximately $16 \%$ of women following menopause [1].

A functional ovarian cyst is a narrow, unilateral, thin-walled, echogenic bag approximately 2,5-6 cm long. A mature graffiti follicle is a sac that develops during ovulation on the surface of a female ovary. Cysts may be categorised as a functional or follicular cyst, depending on the variation of the regular menstrual cycle [2].

There are many kinds of cysts:

The most frequent form of ovarian cyst, the follicular cyst. A follicle holding the ovum (unfertilized egg) will burst during ovulation in menstrual women. If this does not happen, a follicular cyst with a diameter of more than $2.5 \mathrm{~cm}$ may develop.

After ovulation, corpus luteum cysts develop. After the ovum moves to the fallopian tubes, the corpus luteum is the remaining of the follicle. This usually degrades in 5-9 days. A greater than $3 \mathrm{~cm}$ corpus luteum is classified as cystic.

Theca lutein cysts form in the thecal cell layer around the oocytes that mature. Thecal cells may multiply and become cystic under the impact of high HCG. This typically applies to both ovaries [3].
Ovarian functional cysts are a frequent gynaecological issue in reproductive women throughout the globe. These cysts may need surgeries when big, persistent or painful, and may end in the removal of the ovary. Because of the decreased frequency of functional ovarian cysts in early oral contraceptives, many prior research have shown that the use of oral contraception tablets is linked to a lower risk of functional ovary cysts. However, few research have examined the impact of therapy for functioning ovarian cysts by oral contraceptive tablets. Gynecologists treat functional ovarian cysts in contemporary clinical practise with either oral contraceptive tablets or expectant management. Only a few recent studies in comparison with observations only utilise low-dose oral contraceptive pills. Many doctors have begun treating these cysts with birth control tablets to help them leave quicker [4].

Ovarian kysts are often diagnosed with either ultrasound, CT scan or MRI and, if applicable, are linked with clinical and endocrinological testing. Interviews, physical examination and traditional female bimanual pelvic examination are the most essential preparatory procedures for ovary cysts which, together with imaging methods, enable accurate diagnosis. The widely used and cheap technique of visualising women reproductive organs is ultrasound (USG). Magnetic resonance imaging (MR), CT and positron emission tomography (PET) may also be done for malignant lesions [5].

In our nation of Egypt, many ovarian kysts may have operation that is functional or hemorrhagic since ultrasonography equipment are available in every private clinic. In the attempt to minimise this unnecessary operation, our strategy for managing various kinds of cysts is thus recommended. 
The objective of the research was to find out whether or not combination oral contraceptive tablets were necessary for the treatment of functional ovarian kysts in women of reproductive age.

\section{Patients and methods}

Double blinded placebo randomised controlled clinical trials were performed between May 2017 and May 2018 to evaluate the effectiveness of combination oral contraceptive tablets in ovarian cyst therapy. Prior to the study's commencement, approval was acquired from the research ethical committee and head of the Department of Obstetrics and Gynecology at the Benha University Hospital.

Population study and criteria of eligibility

Women who came for various complaints as regards the issue of infertility or the technique of contraception were included in the research. After menstruation, patients were treated to the outpatient clinic where a transvaginal ultrasound was performed to identify instances as follows:

The criteria of inclusion include women of reproductive age, unilateral ovarian cysts, 2.5-6 centimetres in diameter, thin wall, unilocular without internal and solid components.

Premenarchy, postmenopause and neoplastic ovarian edoema contain exclusion criteria (any swelling more than $6 \mathrm{~cm}$ or multilocular).

The patients were split into two equal groups and randomly managed in two ways. Each group consists of 59 women: -

- Group A: women who have been carefully controlled.

- Group B: women receiving combination oral pills.

The research coordinator asked the ladies whether they were willing to have a researcher talk about the project. If so, the investigator talked to them and explained them (risks/benefits, voluntary involvement, processes). Women were given sufficient time to think on the material, were offered questions and free and voluntary permission. Women were then given informed written permission.

\subsection{Intervention study}

In order to assess the size and location of the ovarian cyst (follicular), women were managed by either method, followed by a transvaginal ultrasound every month for three months, and to compare the effect on different modes of management, whether remission, regression, progression or persistence of functional ovarian cyst.

The ladies were randomly allocated either to take oral contraceptive tablets or to take placebo as a control group. The 2 groups were similarly treated and monitored. The study group received oral contraceptive tablets as follows:

Women got a packet of oral contraception (triocept of levenorgestrel 150 microgram + ethinyl estradiol 30 microgram) and were educated about potential adverse effects of oral contraception. A diary card for documenting oral contraception was also supplied to be returned to the doctor for the following cycle. A rendezvous for women in this group was planned for the second ultrasound treatment for one month. If the ovarian cyst does not demonstrate remission, women have been treated equally and transvaginal ultra-sound followed in another month. If the ovarian cyst continues or continues in the second month, the women are monitored during the third month. All findings were compared between the two groups. Data have been gathered and processed using the usual statistical technique.

\subsection{The following results have been evaluated}

Primary results: Remission: the primary result measure and described as an ultrasound test which is not able to identify an ovarian cyst or cyst of less than 2 centimetres in larger size.

\subsection{Secondary results}

1. Regression: Ultrasound examination has been described as the detection of the same ovarian cyst of over 2 centimetres but with more than 50 percent of the pretreatment measures of the greatest dimension.

2. Progression: Ultrasound tests were designed to identify the same ovarian cyst of the greatest size with more than 25 per cent rise in pretreatment measurement.

3. Persistence: The ultrasound test was designed to identify the same ovarian cyst with or without the greatest dimension of less than $50 \%$, or a rise in the greatest dimension of less than $25 \%$ of pretreatment measurements [6].

All pertinent main morbidities were documented which included the main results on the day of the randomization. Repeated clinical and ultrasound tests were conducted monthly for a period of three months following each operation, at the ambulatory clinic of the Department of Obstetrics and Gynecology.

\subsection{Calculation of sample size}

The differential between groups was determined using a sample size of 118 female patients with a twotail $\alpha$ of 0.05 and a (1- $\beta$ ) of 0.80 and by a comparison of 2 independent proportions with a ratio of odd's of 6.3. Our original sample size estimate contained a 10 percent assumption of non-compliance. Example size has been determined using OpenEpi, Version 3, randomised control open source calculator.

\subsection{Randomization}

All women who were present at the outpatient clinic and followed the inclusion criteria were requested to participate in the study by the research coordinator. The approved women were assigned randomly to either the study or control group until the predetermined sample dimension was reached using the block randomization technique. 


\subsection{Blinding}

After assignment, the treatment participants and the study coordinator were blinded. Oral contraceptive tablets and placebo have been placed into 2 distinct colours packets and the treatment type used in each group has only been known to the primary researcher. Unbinding is only permitted if any participant has severe morbidity or even death.

\subsection{Statistical analysis}

Recorded data were analyzed using the statistical package for social sciences, version 20.0 (SPSS Inc., Chicago, Illinois, USA). Quantitative data were expressed as mean \pm standard deviation (SD). Qualitative data were expressed as frequency and percentage. The following tests were done: Independent-samples t-test of significance was used when comparing between two means. Chi-square $\left(\mathrm{x}^{2}\right)$ test of significance was used in order to compare proportions between qualitative parameters. Fisher's exact test: was used to examine the relationship between two qualitative variables when the expected count is less than 5 in more than $20 \%$ of cells. The confidence interval was set to $95 \%$ and the margin of error accepted was set to $5 \%$. So, the p-value was considered significant as the following: Probability (Pvalue), P-value $<0.05$ was considered significant.

\section{Results}

Analysis of data at the end of the study revealed that the age in group (A) with a mean \pm standard deviation (S.D) of $26.3 \pm 2.1$ years, the age in group (B) with a mean \pm S.D of $27.1 \pm 2.7$ (Table 1). Statistical analysis of these data revealed no significant difference among the two groups with respect to the age $(\mathrm{P}$-value $=0.143)$.

After one month, the cysts were found to have remission in 31 of the 40 women in group (A)and 38 of the 40 women in group (B). 19 women in group (A) and 9 women in group (B) showed persistence. None of women in expectant group and 3 women in group (B) shows regression. 9 women in group (A) and 9 women in group (B) show progression. None of the ultrasonic findings was statistically significant. (Pvalue $=0.137)$. fig. (1)

After 2 months in the two study groups. The cysts were found to have remission in 40 women in group (A) and 43 women in group (B). 19 women in group (A) and 16 women in group (B) showed persistence. None of women in group (A) and none of women in group (B) show regression. None of women in group (A) and none of women in group (B) show progression. None of the outcomes was statistically significant. (P-value=0.057). fig (2)

Table (1) Comparison between groups according to age (years).

\begin{tabular}{lcccc}
\hline Age (yr) & $\begin{array}{c}\text { Group (A) } \\
\text { Expectant Group }(\mathbf{n = 5 9 )}\end{array}$ & $\begin{array}{c}\text { Group (B) COC } \\
\text { Group (n=59) }\end{array}$ & t-test & p-value \\
\hline Mean \pm SD & $27.62 \pm 2.21$ & $28.46 \pm 2.84$ & 1.557 & 0.157 \\
\hline
\end{tabular}

Data are presented as mean (SD).Unpaired t test.

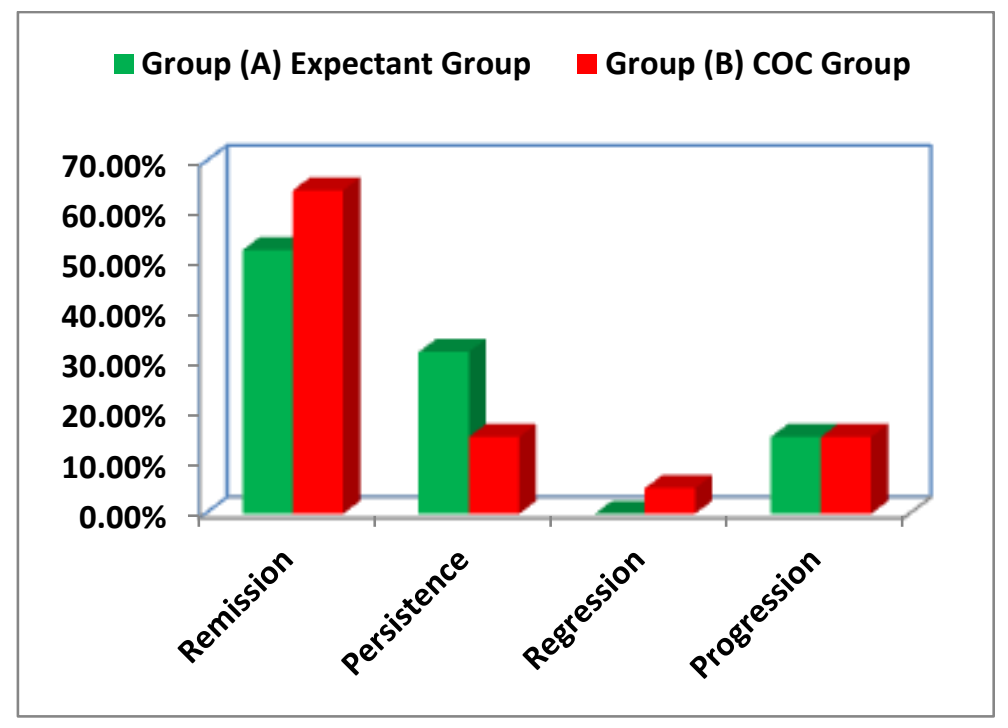

Fig. (1) Bar chart between groups according to outcome of management aftermonth. 


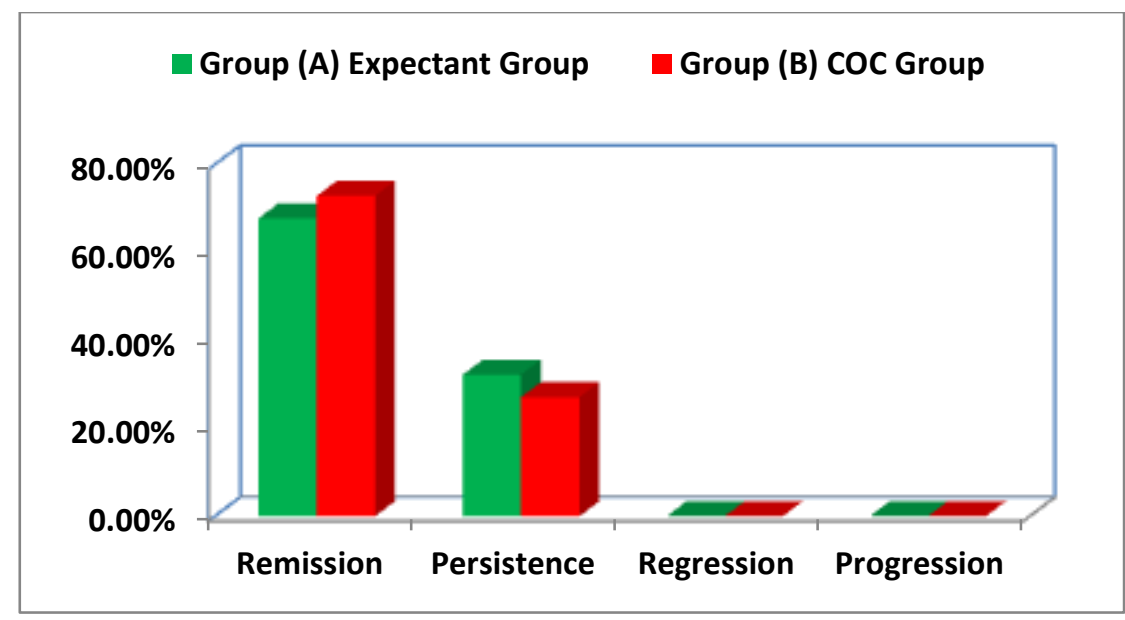

Fig. (2) Bar chart between groups according to outcome at 2 months.

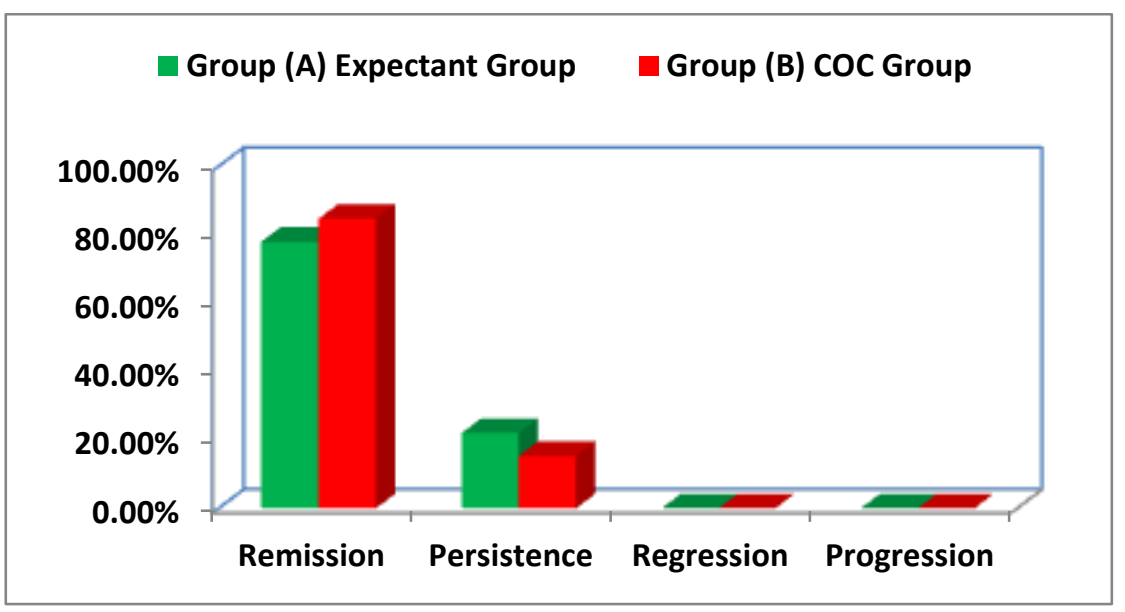

Fig. (3) Bar chart between groups according to outcome after 3 months.

After 3 months in the two study groups. The cysts were found to have remission in 46 women in group (A) and 50 women in group (B). 13 women in group (A) and 9 women in group (B) showed persistence. None of women in group (A) and (B) show regression. None of women in groups and (B) showed progression. None of the outcome was statistically significant (P-value=0.493). fig. (3)

\section{Discussion}

The primary purpose of the prospective randomised controlled study was to evaluate various methods of managing functional ovarian kysts by comparing spontaneously occurring functional ovarian kyst remission rates at 1,2 and 3 months between the treatment of combined oral contraceptives (gruppe B) and the expectant management (gruppe A). 118 participants were assigned randomly for each group in our research.

The percentages of functional ovarian cyst remits were as follows: $52.5 \%$ and $64.4 \%$ in Groups A and B correspondingly after 1 month.

After 2 months, $67.8 \%$ and $72.9 \%$ respectively in Groups A and B
After 3 months, in Groups A and B, 78.0\% and $84.7 \%$ respectively

Thirty-six patients with functional ovarian cyst have been randomised to expectant treatment (groop I, $\mathrm{n}=18$ ) and oral contraceptives (group II, $\mathrm{n}=18$ ), in a comparable research by Aylin et al. (2008). Following one cycle of treatment, cyst clearance was seen in $44.4 \%(n=8)$ and $55.5 \%(n=10)$, of the 18 patients respectively in group I and in group II. The total cyst resolution in Group I was $66.6 \%(\mathrm{n}=12)$ while in Group II it was $72.2 \% \quad(n=13)$. There was no statistically significant difference in any cycle between the two groups.

Another prospective research comparable to that performed by $\mathrm{Naz}$ et al. [7]. in Aylin et al. [8], comprised 47 individuals with functioning ovarian cysts. Twenty-two (46.80 per cent) waitant management (Group A) and oral contraceptives (Group B) patients were advised in 25 patients (53.19 percent ). After 2 months of ultrasonography, the cyst resolution was 72.72 percent in Group A and 80.0 percent in Group B. In the two groups, there was no statistically significant difference in cyst resolution. These findings were in line with our research. 
Mackenna et al. [9] had a randomised controlled study evaluating the effects of combination oral contraceptive tablets on the clearance of functional ovarian cysts compared with expectant care. In addition, after 2 months, all the remaining cysts were resolved. These findings were agreed with our research.

Turan et al.[10] demonstrated that remission rates of spontaneously developed functional cyst in 80 women of their reproductive age were comparable to expectant treatment, both at 5 and 10 weeks of oral contraceptive treatment. With five weeks of treatment, 88.9 percent of women taking monophasic oral contraceptive tablets and 76 percent of women with expected management vanished from the functional ovarian cyst. The disappearance rates were more comparable after 10 weeks of treatment, $100 \%$ and 94.1 percent, respectively. An inadequate number of individuals included in the research was ascribed to the lack of statistical significance.

Our results are consistent with Kilicdge et al. [11], which included a limited number of patients for each group and showed that the remission rate of functional ovarian cyst compared to expectant management of oral contraceptive tablets was substantial.

Our results also accord with the Sanersak et al randomised controlled study [6]. There were 70 women in this research. The remission rates in the combination oral contraceptive group after 2 months were $24 \backslash 33$ (72.2 percent) and in the expectant group $23 \backslash 34$ (67.6 percent). No statistically significant differences existed.

Our findings agree with the Taskin and Young [12] randomised controlled trial of combined oral contraceptives versus waiting for functional ovarian cyst management which examined the efficacy of the studies, which comprised 25 women in the oral contraceptive group, and 20 women in the expectant group. Remission rates after 3 months were $13 \backslash 25$ $(52 \%)$ and $10 \backslash 20(50 \%)$ in the expectant group in combination oral contraceptive groups, and significant differences were not seen.

Our findings are also in agreement with Ghasemi et al. [13] where patients have undergone a two-year transvaginal ultrasound follow-up protocol, 38.5 percent were resolved spontaneously, 25.5 percent continued without change for cysts less than $80 \mathrm{~mm}$, and 20.8 percent were operatively removed.

Our results are in line with the study of Abdulabbar et al. [14] of 244 instances of ovarian cysts that revealed no advantage for the clearance of ovarian cysts in the first and second cycles of hormonal treatment.

Our findings agree with Pascual et al. [15] that 31.8 percent of all females underwent surgery during the follow-up to 408 females with ovarian cysts due to physical discomfort and cyst torsion. The authors in this research contrasted hormonal treatment with observation; in the first cycle 56 percent improved, but in the medication group a greater percentage was improved $(\mathrm{P}=0.087)$. Ovarian cysts in both groups were either solved in two cycles of waitant management (Group A) or oral contraception (Group B) regardless of the diameter of the cyst, lower abdominal pressure and internal echogenic cysts.

Therefore, medications for individuals with functioning ovarian cysts appear to be unneeded.

\section{Conclusion}

Functional ovarian cysts are common, frequently asymptomatic and often resolve spontaneously. There is no proven advantage in the use of combined oral contraceptives.

\section{References}

[1] S.Kiemtoré. "Diagnosis and management of a giant ovarian cyst in the gravid-puerperium period: a case report," BMC Pregnancy Childbirth, vol. 19, pp. 1-7, 2019.

[2] E.K.Ross and M. Kebria, "Incidental ovarian cysts: When to reassure, when to reassess, when to refer.," Cleve. Clin. J. Med., vol. 80, pp. 503-514, 2013.

[3] R.L.Rosenfield and D.A. Ehrmann, "The pathogenesis of polycystic ovary syndrome (PCOS): the hypothesis of PCOS as functional ovarian hyperandrogenism revisited," Endocr. Rev., vol. 37, pp. 467520, 2016.

[4] M.Tafazoli, E. Fazeli, S.Dadgar, and M.Nematy, "The association of the dietary fat and functional ovarian cysts in women of reproductive age referring to three hospitals in Mashhad, Iran, 2014," Int. J. community based Nurs. midwifery, vol. 4, pp. 148, 2016.

[5] M.Walczewska, A.Mocarska, F.Burdan, M.Janczarek, I.Żelazowska-Cieślińska, and E. Starosławska, "Diagnosis of benign ovarian lesions using imaging techniques," Pol. Merkur. Lek. Organ Pol. Tow. Lek., vol. 38, pp. 55-60, 2015.

[6] S.Sanersak, S.Wattanakumtornkul, and C. Korsakul, "Comparison of low-dose monophasic oral contraceptive pills and expectant management in treatment of functional ovarian cysts," Journal-Medical Assoc. Thail., vol. 89, pp. 741, 2006.

[7] T.Naz, Z.Akhter, and T.Jamal, "Oral contraceptives versus expectant treatment in the management of functional ovarian cysts," J Med Sci, vol. 11, pp. 185-188, 2011.

[8] C.I.L.Ayline Pelin, E.Ozturkoglu, B. Demir, M. Gunes, and A. Haberal, "Is hormonal therapy needed in the management of functional ovarian cyst," Turkey Ankara Etlik Matern. Women's Heal. Teach. Res. Hosp., pp. 1301-3314, 2008.

[9] A.MacKenna, C.Fabres, V.Alam, and V.Morales, "Clinical Management of Functional Ovarian Cysts: A Prospective and 
Randomized Study," Obstet. Gynecol. Surv., vol. 56, pp. 281-282, 2001.

[10] C.Turan, C.G.Zorlu, M. Uḡur, T. Özcan, B. Kaleli, and O. Gökmen, "Expectant management of functional ovarian cysts: an alternative to hormonal therapy," Int. J. Gynecol. Obstet., vol. 47, pp. 257-260, 1994.

[11]E.B.Kilicdag, E. Tarim, S. Erkanli, E. Aslan, G. Asik, and T. Bagis, "How effective are ultra-low dose oral contraceptive pills for treatment of benign ovarian cysts?," Fertil. Steril., vol. 80, pp. 218-219, 2003.

[12] O.TASKIN, D.C.YOUNG, R. MANGAL, and I. ARUH, "Prevention and treatment of ovarian cysts with oral contraceptives: a prospective randomized study," J. Gynecol. Surg., vol. 12, pp. 21-24, 1996.

[13]F.Ghasemi, S.Mirzaee, and P.Amini, "Comparison of Hormone Therapy with Expectant Management in the Clinical Management of Functional Ovarian Cysts: A Randomized Clinical Trial," Adv. Nurs. Midwifery, vol. 28, pp. 48-54, 2018.

[14] H.S.Abduljabbar et al., "Review of 244 cases of ovarian cysts," Saudi Med. J., vol. 36, pp. $834,2015$.

[15] M.A.Pascual. "Long-term results for expectant management of ultrasonographically diagnosed benign ovarian teratomas," Obstet. Gynecol., vol. 130, pp. 1244-1250, 2017. 